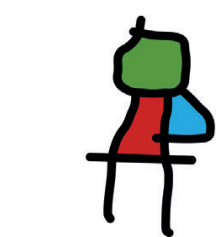

SEICAIP

\section{Allergologia et immunopathologia}

Sociedad Española de Inmunología Clínica, Alergología y Asma Pediátrica

www.all-imm.com

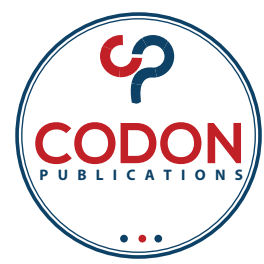

ORIGINAL ARTICLE

OPEN ACCESS C(1) (9)

\title{
Abnormalities in subsets of $B$ and $T$ cells in Mexican patients with inborn errors of propionate metabolism: observations from a single-center case series
}

\author{
E.A. Medina-Torres ${ }^{a \dagger}$, M. Vela-Amieva ${ }^{\mathrm{b} \dagger}$, L. Galindo-Campos ${ }^{\mathrm{b}, \mathrm{c}}$, I. Ibarra-González ${ }^{\mathrm{d}}$, \\ S. Espinosa-Padilla ${ }^{\mathrm{a}}$, S. Guillén-López ${ }^{\mathrm{b}}$, L. López-Mejía ${ }^{\mathrm{b}}, \mathrm{C}$. Fernández-Lainez ${ }^{\mathrm{b} *}$ \\ aUnidad de Investigación en Inmunodeficiencias, Instituto Nacional de Pediatría, Secretaría de Salud, México \\ 'Laboratorio de Errores Innatos del Metabolismo y Tamiz, Instituto Nacional de Pediatría, Secretaría de Salud, México \\ 'Alumno Tesista de la Facultad de Química de la Universidad Nacional Autónoma de México, UNAM, México \\ ¿Unidad de Genética de la Nutrición, Instituto de Investigaciones Biomédicas-Instituto Nacional de Pediatría, UNAM, México \\ ${ }^{+}$Both authors contributed equally to the manuscript.
}

Received 17 February 2020; Accepted 19 May 2020

Available online 2 January 2021

\section{KEYWORDS}

immunodeficiency;

inborn errors of

metabolism;

inherited metabolic

disorders;

methylmalonic

acidemia; propionic acidemia.

\begin{abstract}
Background: Propionate inborn errors of metabolism (PIEM), including propionic (PA) and methylmalonic (MMA) acidemias, are inherited metabolic diseases characterized by toxic accumulation of propionic, 3-hydroxypropionic, methylcitric, and methylmalonic organic acids in biological fluids, causing recurrent acute metabolic acidosis events and encephalopathy, which can lead to fatal outcomes if managed inadequately. PIEM patients can develop hematological abnormalities and immunodeficiency, either as part of the initial clinical presentation or as chronic complications. The origin and characteristics of these abnormalities have been studied poorly. Thus, the aim of the present work was to evaluate and describe lymphoid, myeloid, and erythroid cell population profiles in a group of clinically stable PIEM patients.

Methods: This was a retrospective study of 11 nonrelated Mexican PIEM patients. Clinical, biochemical, nutritional, hematological, and lymphocyte subsets were analyzed.

Results: Despite being considered clinically stable, $91 \%$ of patients had hematological or immunological abnormalities. The absolute lymphocyte subset counts were low in all patients but one, with CD4+ T-cell lymphopenia, being the most common one. Furthermore, of the 11 studied subjects, nine presented with a low CD4/CD8 ratio. Among the observed hematological alterations, bicytopenia was the most common (82\%) one, followed by anemia (27\%).

Conclusion: Our results contribute to the landscape of immunological abnormalities observed previously in PIEM patients; these abnormalities can become a life-threatening chronic complications because of the increased risk of opportunistic diseases. These findings allow us to propose the inclusion of monitoring immune biomarkers, such as subsets of lymphocytes in the follow up of PIEM patients.
\end{abstract}

๑) 2021 Codon Publications. Published by Codon Publications.

*Corresponding author: Cynthia Fernández-Lainez. Laboratorio de Errores Innatos del Metabolismo y Tamiz, Instituto Nacional de Pediatría, Torre de Investigación, Av. IMAN \#1, piso 9, Alcaldía Coyoacán, CDMX, CP 04530. Email address: lainezcynthia@hotmail.com 


\section{Introduction}

Propionate inborn errors of metabolism (PIEM), including propionic (PA) and methylmalonic (MMA) acidemias, are low-prevalence monogenic diseases (1:50,000-1:80,000 newborns) that cause dysfunctioning of enzymes propionyl-CoA carboxylase (PCC) and methylmalonyl-CoA mutase (MUT), respectively. ${ }^{1}$ These disorders are biochemically characterized by the accumulation of propionic, 3-hydroxypropionic, methylcitric, and methylmalonic organic acids, as well as propionylcarnitine, propionylglycine, tiglylglycine, and glycine, in biological fluids (Figure 1).

Patients with PA and MMA have similar clinical and biochemical pictures, mainly characterized by encephalopathy, hypotonia, seizures, movement disorders, optic atrophy, developmental delay, neuropsychiatric symptoms, renal failure, and cardiomyopathy, among others, and accompanied by recurrent ketoacidosis crisis, hyperammonemia, hypoglycemia, hyperlactatemia, and hyperuricemia. Severity of disease is related to the residual enzymatic activity determined by the pathogenic gene variant, age at onset of symptoms, age at starting of treatment, and treatment compliance. ${ }^{1}$ The therapeutic approach to these disorders is based on the dietary restriction of propionyl-CoA precursors (Figure 1) accompanied by pharmacological treatment such as ammonia scavenger drugs, carnitine, and vitamins. ${ }^{1}$ Liver transplant also has been used, especially for patients with frequent metabolic decompensations. ${ }^{2}$ In spite of the type of treatment, the outcome of PIEM is suboptimal, and these patients can develop different complications in the central nervous, renal, cardiologic, and hematopoietic systems that might lead to death. ${ }^{1}$

Hematological abnormalities, including anemia, neutropenia, thrombocytopenia, and pancytopenia, have been described in PIEM patients on initial presentation, in periods of acute metabolic decompensations (AMD), or as a lifelong complication,,$^{3,4}$ and these have been described more often in PA patients than in those with MMA. ${ }^{4}$ Bone marrow abnormalities have also been reported, including medullary hypoplasia, dysmyelopoiesis, hemophagocytosis, numerous histiocytes, and multinucleated megakaryocytes. ${ }^{3,5}$ Furthermore, the presence of immunodeficiency requiring chronic treatment has been reported in PIEM patients. ${ }^{6}$ Moreover, infections and sepsis due to diverse types of pathogens could also exist. ${ }^{7}$ The origin, characteristics, and pathophysiology of hematological and immunological alterations in these patients remain elusive. In the most recent PIEM management guidelines, there is no outright recommendation to consider these patients as immunodeficient. ${ }^{1}$ Although there are some reports on the presence of abnormal lymphocyte counts in patients with PIEM, ${ }^{8,9}$ the characteristics of lymphocytic subsets in these patients are not known adequately. The aim of this work is to evaluate and describe lymphoid, myeloid, and erythroid cell population profiles in a group of patients with PIEM.
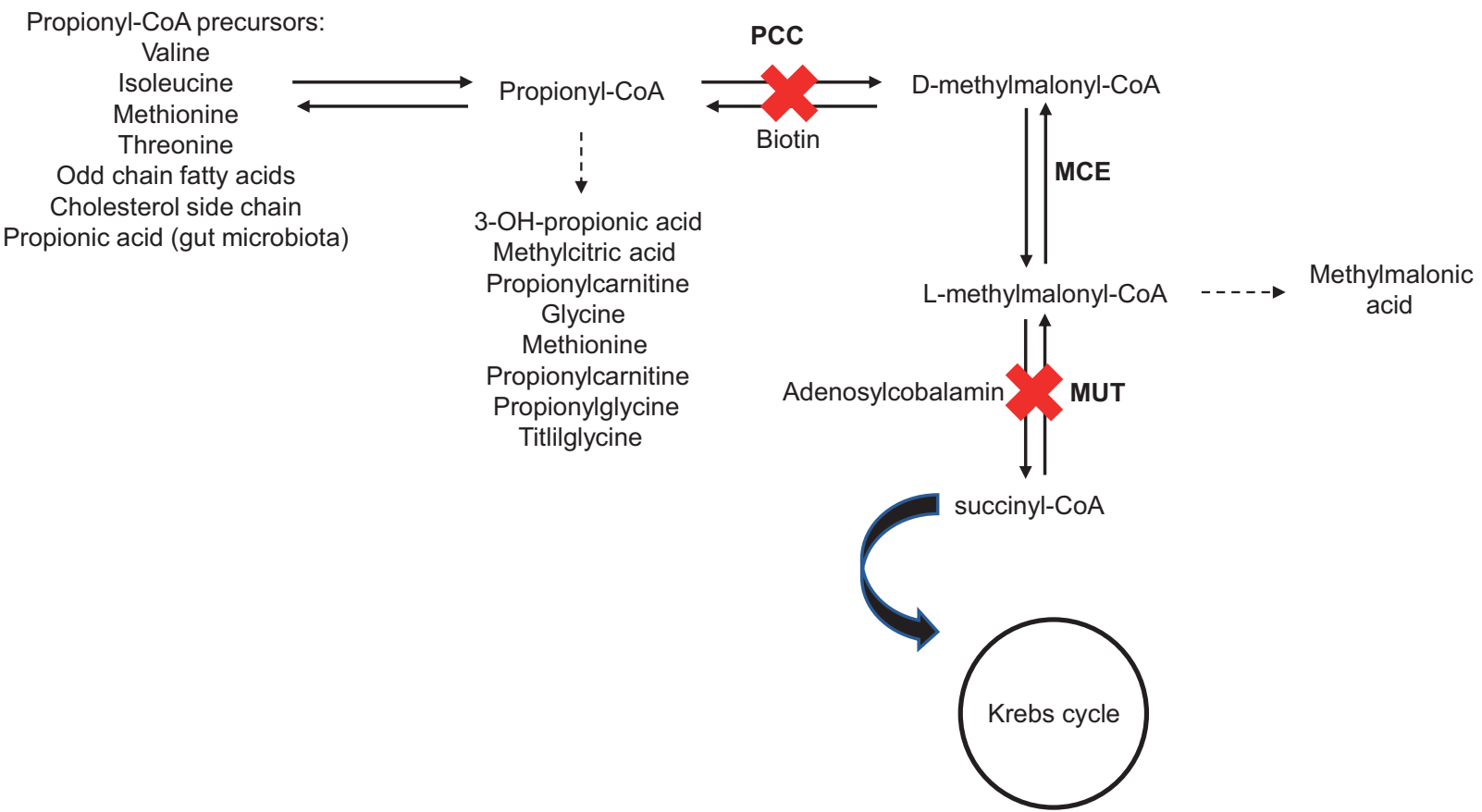

Figure 1 Metabolic pathway of propionate. Dotted arrows denote the accumulation of toxic metabolites due to the activation of alternate pathways because of the enzymatic block (red X) in the case of PA and MMA occurrence. Differential diagnosis of propionic acidemia: Elevation of 3-OH-propionic acid, methylcitric acid, propionylcarnitine, glycine, methionine, propionylcarnitine, propionylglycine and titlilglycine; in the case of methylmalonic acidemia, same metabolites can be elevated accompanied by methylmalonic acid. Abbreviations: PCC: propionyl-CoA carboxylase; MCE: Methylmalonyl-CoA epimerase; MUT: Methylmalonyl-CoA mutase. 


\section{Methods}

This was a retrospective study of patients who attended a pediatric tertiary care center in Mexico City that includes an inborn error of metabolism unit and an immunodeficiency national reference research unit.

\section{Subjects}

Data from the medical files of 11 Mexican unrelated children with PIEM (five males and six females; age ranging from 11 months to 11 years ) were analyzed. MMA and PA diagnosis was established previously in five and six subjects, respectively, by quantifying blood amino acids and acylcarnitines through tandem mass spectrometry (MS/MS), and the diagnoses were confirmed by the analysis of urinary acid profile with gas chromatography-mass spectrometry. For the differential diagnosis of MMA, plasma homocysteine was quantified by high-performance liquid chromatography coupled to fluorescence detection; all these techniques were performed according to the methodology reported previously. ${ }^{10}$ Patients had nutritional, medical, and biochemical follow-up of 10 months to 10 years, which included anthropometric measurements, hemograms, blood amino acids, acylcarnitines, and ammonia determination. Clinically stable patients were defined as those seen at the outpatient clinic without presenting any symptoms of infection or metabolic distress at the time of their evaluation.

\section{Lymphocyte subsets by flow cytometry}

Blood samples for the analysis of lymphocyte subsets were taken simultaneously for routine outpatient follow-up. In a sample of $50-\mu \mathrm{L}$ whole blood, staining for superficial antigens for $T$ cells (CD3PerCP/CD4FITC/CD8 PE), B cells (CD3FITC/CD19PE/CD45PerCP), and natural killer (NK) cells (CD3FITC/CD45PerCP/CD16+56PE) was performed with Multitest reagents (Becton Dickinson, San Jose, CA, USA). For this purpose, blood samples were incubated for 30 min at room temperature with $10 \mu \mathrm{L}$ of each reagent, followed by lysis of erythrocytes with $500 \mu \mathrm{L}$ of BD-FACS lysis solution (Becton Dickinson, San Jose, CA, USA) and subsequent analysis in a FACS Aria 1 flow cytometer (Becton Dickinson, San Jose, CA, USA).

\section{Amino acids and acylcarnitine analysis}

In one drop of capillary blood, amino acids, free carnitine, and acylcarnitines were measured using MS/MS equipment with a commercial kit (NeoBase non-derivatized MS/MS kit; PerkinElmer Waltham, MA, USA) according to the methodology reported previously..$^{10}$

\section{Ethics}

This study was carried out in accordance with the ethical and legal disposition of the National Pediatrics Institute and according to the Helsinki Declaration. The study was approved by the institutional research and ethics committees (Project 052/2016). Written informed consent was obtained from parents of each patient.

\section{Results}

The clinical and biochemical characteristics of PIEM patients, categorized by subgroup of disease (PA or MMA), are described in Table 1. On a routine hemogram, three patients had neutropenia (patients $B, C$, and 6), and one had lymphopenia (patient 3). No abnormalities were observed in monocytes, eosinophils, or basophils. Nine patients (82\%) had abnormal red cells, white cells, or platelet values, with red and white cell counts being affected most frequently (Table 1). Low levels of branched chain amino acids (valine, leucine, and isoleucine) were found as the most common biochemical abnormalities (5/11, 45.4\%), and in the acylcarnitine profile, propionylcarnitine was elevated in all patients and the ammonia level was high in two patients (Table 1). On the other hand, all MMA patients presented with normal homocysteine levels. At the nutritional level, the body mass index (BMI) Z-score results showed that the majority of patients were eutrophic (7/11, $64 \%$ ); one was overweight, another had obesity (patients 2 and $A$ ), and patients $C$ and 6 had moderate and severe malnutrition, respectively $(2 / 11,18 \%)$. Regarding $T$ and $B$ cell subsets, the CD4+ T-lymphocyte absolute count was below the reference value for all studied patients except one, and $7 / 11$ patients had low levels of CD8+ T cells. On the other hand, 7/11 patients presented with low counts of CD19+ B cells. Conversely, natural killer cells $(C D 16+56+)$ were low only in 2/11 patients (Table 2).

\section{Discussion}

Patients with PIEM, as demonstrated in our work, have alterations in the absolute counts of $B$ or $T$ cell subsets and are at risk of developing immunodeficiency. Our results, which have the strength of having been obtained in the outpatient clinic, contribute to the landscape of immunological abnormalities observed previously in patients with PIEM.6,9

A high number of patients with hematological abnormalities were found (9/11, 82\%); bicytopenia was the most frequent abnormality (4/11), followed by anemia (3/11, $27 \%$ patients), lymphopenia $(1 / 11,9 \%)$, and pancytopenia $(1 / 11$, 9\%) (Table 1). Depletion of lymphocyte subset was observed in all studied patients, with the most common being low counts for the CD4 subsets (Table 2), which was present in all patients except one (patient 6; Table 2). Additionally, CD8 was low in 7/11 (63\%), CD19 in 7/11 (64\%), and CD16+56 in $2 / 11(18 \%)$ patients, which contrast with the number of white cell abnormalities in routine hemograms (found in only 4/11, 36\% patients; Table 1). T-cell CD4+ lymphopenia should be considered by physicians as an aspect of monitoring patients with PIEM. This condition raises the risk of opportunistic diseases,11 and has been associated with sepsis in infected hospitalized patients. Furthermore, our findings support the relevance of including the lymphocytic subset counts as potential prognostic biomarkers and 


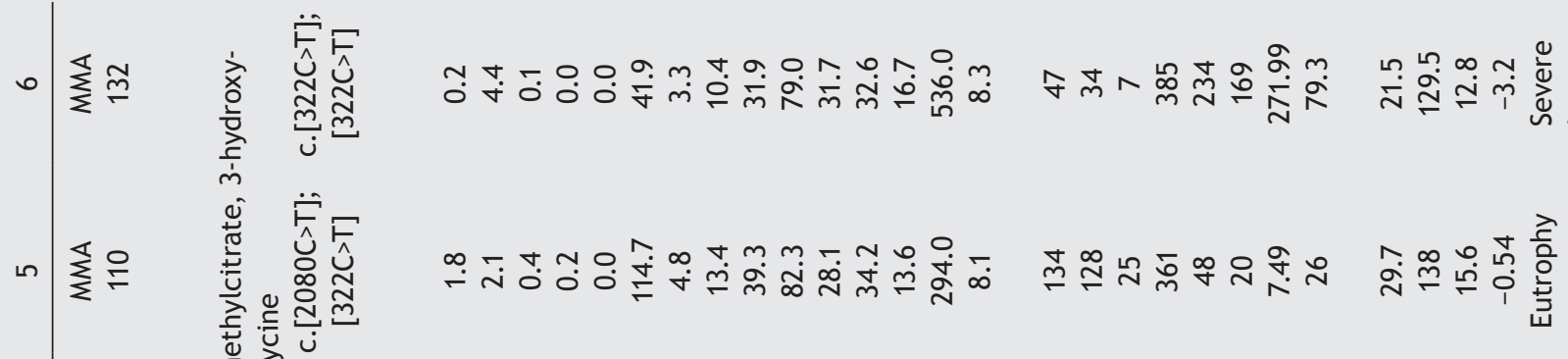
$\varepsilon$ के +

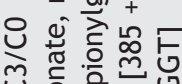

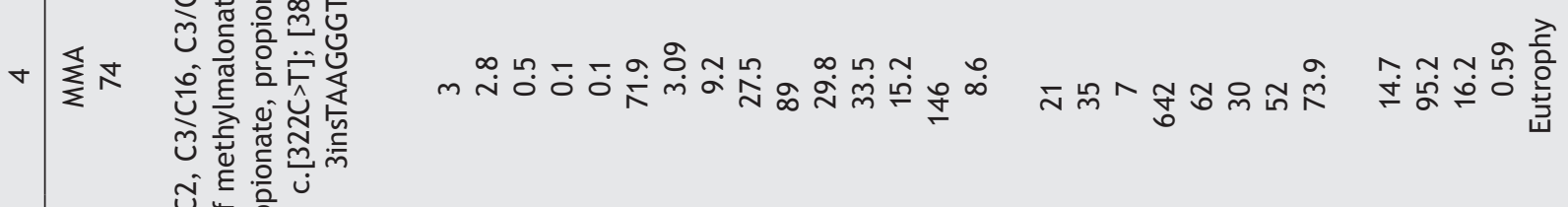

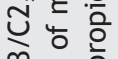

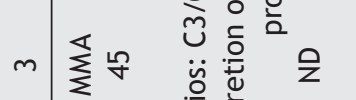

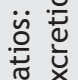

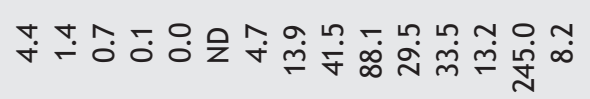

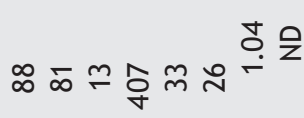

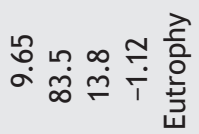

등

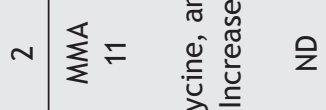

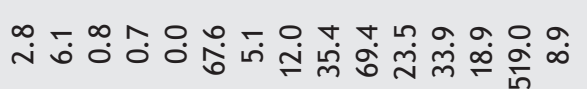

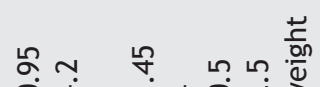

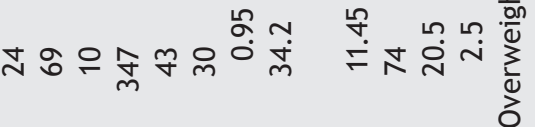

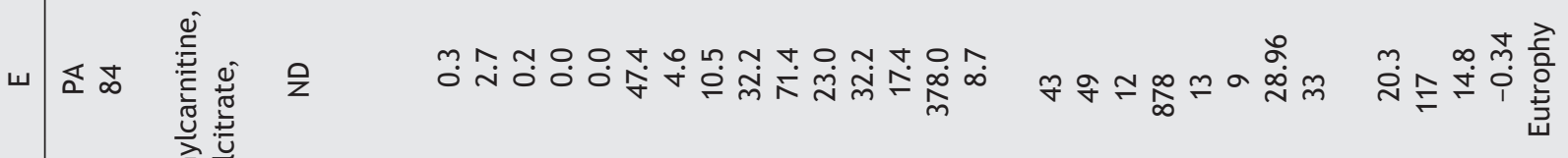

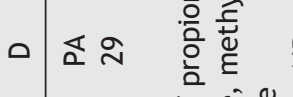

谙

垔.

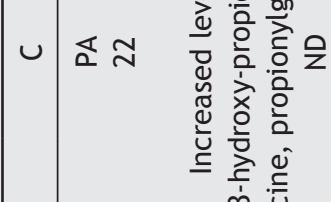

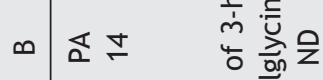

돌 항

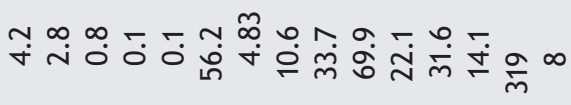
ห+ำ

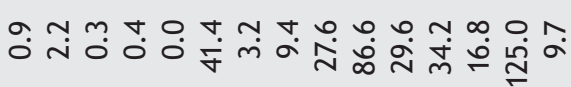

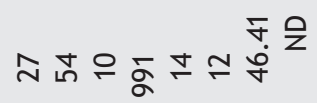

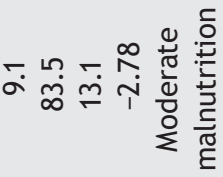
F N

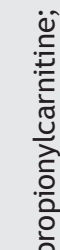
î

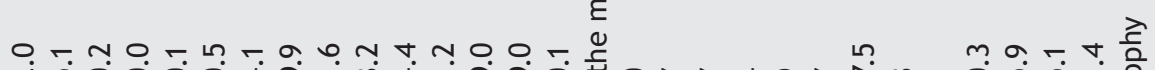

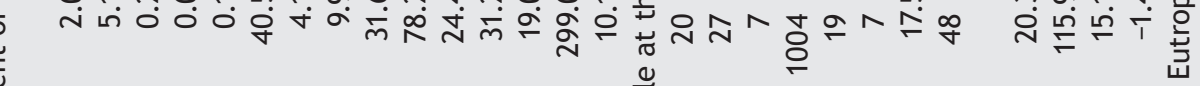

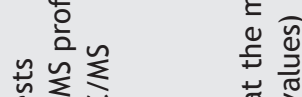

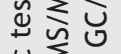

$\because \sum \frac{0}{\bar{y}} \geq$

突

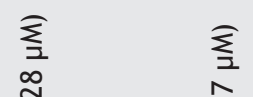

¿ ठ

$\frac{\widehat{\Phi}}{\frac{\widehat{\Phi}}{\sqrt{0}}}$

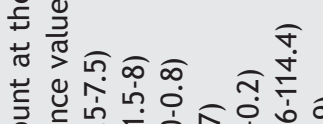

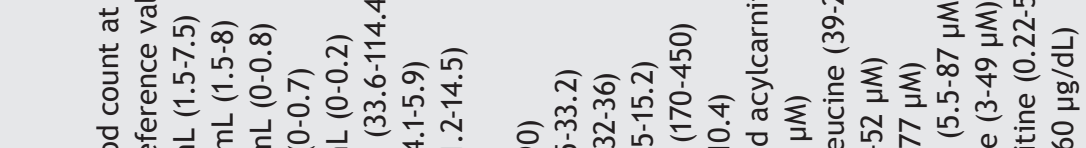

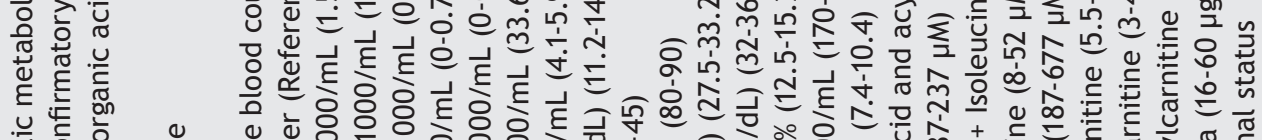


Table 2 Absolute count of lymphocytic subsets and its interpretation of PIEM patients.

\begin{tabular}{|c|c|c|c|c|c|c|c|c|c|c|c|c|c|c|}
\hline $\begin{array}{l}\text { Patitent } \\
\text { ID }\end{array}$ & Diagnosis & $\begin{array}{c}\text { Age } \\
\text { (months) }\end{array}$ & $\begin{array}{l}\text { CD4 } \\
\text { Abs }\end{array}$ & CD4 Ref & Inter & CD8 Abs & CD8 Ref & Inter & $\begin{array}{c}\text { CD19 } \\
\text { Abs }\end{array}$ & CD19 Ref & Inter & $\begin{array}{c}\text { CD16+ } \\
56 \\
\text { Abs }\end{array}$ & $\begin{array}{l}\text { CD16+ } \\
56 \text { Ref }\end{array}$ & Inter \\
\hline $\mathrm{F}$ & PA & 11 & 783 & $1400-4300$ & $\downarrow$ & 809 & $500-1700$ & $\mathrm{~N}$ & 262 & $610-2500$ & $\downarrow$ & 396 & $160-950$ & $\mathrm{~N}$ \\
\hline A & PA & 14 & 270 & $1300-3400$ & $\downarrow$ & 177 & $620-2000$ & $\downarrow$ & 234 & $720-2600$ & $\downarrow$ & 317 & $180-920$ & $\mathrm{~N}$ \\
\hline B & PA & 14 & 344 & $1300-3400$ & $\downarrow$ & 158 & $620-2000$ & $\downarrow$ & 608 & $720-2600$ & $\downarrow$ & 189 & $180-920$ & $\mathrm{~N}$ \\
\hline C & PA & 22 & 686 & $1300-3400$ & $\downarrow$ & 358 & $620-2000$ & $\downarrow$ & 337 & $720-2600$ & $\downarrow$ & 13 & $180-920$ & $\downarrow$ \\
\hline D & PA & 29 & 246 & $700-2200$ & $\downarrow$ & 189 & $490-1300$ & $\downarrow$ & 615 & $390-1400$ & $\mathrm{~N}$ & 350 & $130-720$ & $\mathrm{~N}$ \\
\hline$E$ & PA & 84 & 629 & $650-1500$ & $\downarrow$ & 589 & $370-1100$ & $\mathrm{~N}$ & 475 & $270-860$ & $\mathrm{~N}$ & 138 & $100-480$ & $\mathrm{~N}$ \\
\hline 2 & MMA & 11 & 946 & $1400-4300$ & $\downarrow$ & 271 & $500-1700$ & $\downarrow$ & 2285 & $610-2500$ & $\mathrm{~N}$ & 486 & $160-950$ & $\mathrm{~N}$ \\
\hline 3 & MMA & 45 & 103 & $700-2200$ & $\downarrow$ & 59 & $490-1300$ & $\downarrow$ & 387 & $390-1400$ & $\downarrow$ & 255 & $130-720$ & $\mathrm{~N}$ \\
\hline 4 & MMA & 74 & 503 & $650-1500$ & $\downarrow$ & 550 & $370-1100$ & $\mathrm{~N}$ & 301 & $270-860$ & $\mathrm{~N}$ & 77 & $100-480$ & $\downarrow$ \\
\hline 5 & MMA & 110 & 329 & $650-1500$ & $\downarrow$ & 330 & $370-1100$ & $\downarrow$ & 232 & $720-2600$ & $\downarrow$ & 128 & $100-480$ & $N$ \\
\hline 6 & MMA & 132 & 842 & $650-1500$ & $\mathrm{~N}$ & 569 & $370-1100$ & $\mathrm{~N}$ & 548 & $720-2600$ & $\downarrow$ & 122 & $100-480$ & $N$ \\
\hline
\end{tabular}

Symbols and abbreviations: PA, propionic acidemia; MMA, methylmalonic acidemia; Abs, absolute count; N, normal; $\downarrow$, low; Inter, interpretation; Ref, reference value

therapeutic targets for the designing of better therapeutic interventions to avoid fatal outcomes. ${ }^{12}$

Hematological and immunological abnormalities in patients with PIEM could be explained by different reasons, some of which could occur even simultaneously. Inoue et al. ${ }^{5}$ studied the direct effect of methylmalonic acid present in the plasma of an MMA patient in vitro. They added it to cultured bone marrow cells, whose growth was seemingly inhibited in those cells that were cultured in the presence of known amounts of methylmalonic acid standard reagent in a dose-dependent manner. These findings suggested the inhibition of bone marrow proliferation by this toxic metabolite. ${ }^{5}$ Moreover, mitochondrial function, which is essential for adequate hematopoiesis, is one of the main targets of oxidative stress caused by abnormal organic acids that accumulate in propionate metabolism defects. Impaired mitochondrial function could produce arrest in the maturation of hematopoietic precursors, inhibition of bone marrow proliferation, and shortening of erythrocyte half-life, thereby leading to the development of cytopenia. ${ }^{3,9}$ A recently published systematic data analysis of cohort studies and case reports on PA and MMA has suggested that mitochondrial impairment could possibly be related to anemia, neutropenia, thrombocytopenia, and pancytopenia, providing evidence for its role in the pathophysiology of complications in patients with PA and MMA. ${ }^{4}$ However, further studies are required to demonstrate whether toxic intermediary metabolites contribute to the pathophysiology of PIEM. The possible influence of nutritional status, which may be particularly related to anemia, was exceeded by the previously described toxic effects of organic acids on lymphoid, myeloid, and erythroid populations, because most of the patients were supplemented with iron and a vitamin and mineral complex. Interestingly, nutritional status did not have any correlation with anemia, amino acids, or absolute count of lymphocytes, as would have been expected, because most of the patients had normal BMI (7/11, 64\%; Table 1).

Despite the evidence of hypoplasia, aplasia, myelodysplastic syndrome, and hemophagocytic lymphohistiocytosis found in PIEM patients, ${ }^{9}$ routine immunological evaluation and bone marrow examination are not a generalized recommendation in the current management of PIEM patients, and the immunological assessment is only required when the patients are hospitalized or when a serious infection is suspected. ${ }^{1}$ One of the limitations of this study is the small number of studied patients; however, the low T- and B-lymphocyte subsets found in a high proportion of studied patients (Table 2) allow us to propose that immunological assessment be considered in the routine follow-up of these patients to achieve more effective therapeutic interventions for reducing morbidity and mortality associated with hematological and immune abnormalities. This would also help improve our understanding of the pathophysiology of bone marrow involvement in these congenital disorders. It would also be very useful to quantify immunoglobulin levels in these patients because when low levels of B- and T-lymphocytes are present, hypogammaglobulinemia could occur, and therefore treatment with human gamma globulin may help in case of sepsis in these patients. A patient with methylmalonic aciduria was described recently when looking for genetic diagnoses in patients with suspected primary immunodeficiency by new-generation sequencing. The patient was a one-month-old male child with pancytopenia, hemophagocytosis, and a clinical suspicion of familial HLH or X-linked lymphoproliferative syndrome; however, a homozygous insertion was detected in one nucleotide of MMACHC gene. ${ }^{13}$ This case and our results in the present work show that we must now think about the importance of differential diagnosis between an inborn error of metabolism, such as PA and MMA acidemias, and an innate error of immunity or primary immunodeficiency.

Further studies are required to obtain a better understanding of the immunological status of PIEM patients, including lymphocyte subset analyses by flow cytometry, immunoglobulin determinations, and bone marrow examinations. This could guide to other therapeutic options to decrease morbidity and mortality because of infectious diseases. 


\section{Acknowledgments}

The authors would like to thank the patients and parents who participated in this study. We also appreciate the technical assistance provided by Aída Jeanette Hernández-Montiel, Ricardo Morales-González, and Jaime Torres-Marcial.

\section{Funding}

This manuscript was supported by the Research Funding of the National Institute of Pediatrics "Recursos Fiscales del Programa E022," Ciudad de México, Mexico.

\section{Conflict of interest}

The authors declare they have no conflicts of interest.

\section{References}

1. Baumgartner MR, Hörster F, Dionisi-Vici C, Haliloglu G, Karall D, Chapman KA, et al. Proposed guidelines for the diagnosis and management of methylmalonic and propionic acidemia. Orphanet J Rare Dis. 2014;9:130. https://doi.org/10.1186/ s13023-014-0130-8

2. Pillai NR, Stroup BM, Poliner A, Rossetti L, Rawls B, Shayota BJ, et al. Liver transplantation in propionic and methylmalonic acidemia: A single center study with literature review. Mol Genet Metab. 2019;128(4):431-443. https://doi.org/10.1016/j. ymgme.2019.11.001

3. Stork LC, Ambruso DR, Wallner SF, Sambrano JE, Moscinski LC, Wilson HL, McCabe ER. Pancytopenia in propionic acidemia: Hematologic evaluation and studies of hematopoiesis in vitro. Pediatr Res. 1986;20(8):783-8. https://doi. org/10.1203/00006450-198608000-00017

4. Haijes HA, Jans JJM, Tas SY, Verhoeven-Duif NM, van Hasselt PM. Pathophysiology of propionic and methylmalonic acidemias. Part 1: Complications. J Inherit Metab Dis. 2019;42(5):730-44. https://doi.org/10.1002/jimd.12129
5. Inoue S, Krieger I, Sarnaik A, Ravindranath Y, Fracassa M, Ottenbreit MJ. Inhibition of bone marrow stem cell growth in vitro by methylmalonic acid: a mechanism for pancytopenia in a patient with methylmalonic acidemia. Pediatr Res. 1981;15(2): 95-8. https://doi.org/10.1203/00006450-198102000-00001

6. Pena L, Burton BK. Survey of health status and complications among propionic acidemia patients. Am J Med Genet A. 2012;158A:1641-6. https://doi.org/10.1002/ajmg.a.35387

7. Al Essa M, Rahbeeni Z, Jumaah S, Joshi S, Al Jishi E, Rashed MS, et al. Infectious complications of propionic acidemia in Saudia Arabia. Clin Genet. 1998;54(1):90-4. https://doi. org/10.1111/j.1399-0004.1998.tb03702.x

8. Griffin TA, Hostoffer RW, Tserng KY, Lebovitz DJ, Hoppel CL, Mosser JL, et al. Parathyroid hormone resistance and $\mathrm{B}$ cell lymphopenia in propionic acidemia. Acta Paediatr. 1996;85(7):875-8. https://doi.org/10.1111/j.1651-2227.1996. tb14172.x

9. Bakshi NA, Al-Anzi T, Mohamed SY, Rahbeeni Z, AlSayed M, Al-Owain M, Sulaiman RA. Spectrum of bone marrow pathology and hematological abnormalities in methylmalonic acidemia. Am J Med Genet A. 2018;176(3):687-91. https://doi. org/10.1002/ajmg.a.38599

10. Ibarra-González I, Fernández-Lainez C, Reyes-González DI, Belmont-Martínez L, Guillén-López S, Monroy-Santoyo S, VelaAmieva M. Inborn errors of intermediary metabolism in critically ill Mexican newborns. JIEMS. 2014;2:1-7. https://doi. org/10.1177/2326409814529649

11. Müller S, Falkenberg N, Mönch E, Jakobs C. Propionic acidaemia and immunodeficiency. Lancet. 1980;1(8167):551-2. https://doi.org/10.1016/S0140-6736(80)92815-9

12. Hohlstein P, Gussen H, Bartneck M, Warzecha KT, Roderburg C, Buendgens $\mathrm{L}$, et al. Prognostic relevance of altered lymphocyte subpopulations in critical illness and sepsis. J Clin Med. 2019;8(3). pii: E353. https://doi.org/10.3390/jcm8030353

13. Rudilla F, Franco-Jarava C, Martínez-Gallo M, Garcia-Prat M, Martín-Nalda A, Rivière $\mathrm{J}$, et al. Expanding the clinical and genetic spectra of primary immunodeficiency-related disorders with clinical exome sequencing: Expected and unexpected findings. Front Immunol. 2019;10:2325. https://doi. org/10.3389/fimmu.2019.02325 